\title{
Abdul H. Sultan, Ranee Thakar, Dee E. Fenner (eds): Perineal and Anal Sphincter Trauma
}

\author{
Springer-Verlag, 2007, ISBN 9781852339265, hardback, 195 pages, $£ 85$
}

\author{
Sharif I.M.F. Ismail
}

Received: 13 October 2008 / Accepted: 26 October 2008 / Published online: 14 November 2008

(C) The International Urogynecological Association 2008

This book provides a comprehensive overview of all aspects related to perineal and anal sphincter trauma, both obstetric and non-obstetric, as evidenced by its multidisciplinary authorship and its diverse content.

It starts by providing a background on embryology and physiological anatomy of the perineum and anal sphincter that describes muscle structure, blood and nerve supply as well as lymphatic drainage to enable understanding of types of tears and how to repair them. The diagnosis of obstetric anal sphincter injuries (OASIS) is discussed with emphasis on proper clinical examination at the time of delivery by midwives and doctors to avoid missing, as well as underdiagnosis of, injuries, and the use of agreed classification. The repair of different grades of tears is described in detail, with evidence-based account of controversial aspects like the type of suture, the technique of repair, after-care, subsequent delivery and preventive measures. The presentation and management of postpartum complications related to perineal and anal sphincter trauma are discussed and the set up of a multidisciplinary one-stop perineal clinic is outlined.

A narrative background idea about female genital modification is given to enable understanding the management of relevant problems in pregnancy and at the time of delivery. Similarly, a background idea about anal continence is provided to enable understanding how anal incontinence reflects more than anatomical disruption at the time of vaginal delivery. This chapter, however, is rather dense and not easy to understand, especially for those not

\section{S. I. Ismail $(\square)$}

Obstetrics and Gynaecology, Yeovil District Hospital,

Higher Kington,

Yeovil, Somerset BA21 4AT, UK

e-mail: sharif212121@yahoo.co.uk involved in colorectal surgery. Paragraphs are long, the technique and value of different investigations are not adequately explained, and no images are provided of the equipment used or the graphs obtained to enable the reader to appreciate what is actually being done. Imaging of the anal sphincter is well outlined, though a more practical approach with more images and diagrammatic explanations would have aided a better grasp of the techniques and their value.

Conservative measures for anal incontinence are discussed. The role of diet, bowel habit re-education, pelvic floor exercises, biofeedback and medication is well described. The value, limitations, and thus place of secondary anal sphincter repair are discussed in practical terms rightly before describing techniques and after-care. In addition, an idea is given about other surgical techniques, including bulking agents, artificial sphincter and sacral nerve stimulation.

The pathology, presentation and management of rectovaginal fistula are covered in a simple practical manner and its relationship to obstetric anal sphincter injuries is highlighted. Similarly, the legal principles and steps relevant to perineal and anal sphincter trauma are explained in a simple and clear manner to enable understanding how to avoid and deal with claims.

The book represents a valuable resource for those who are involved in the management of perineal and anal sphincter trauma to further their knowledge and understanding of this emerging field and advance their skills in patient care. Given its scope and coverage of multidisciplinary aspects, it is suitable for obstetricians, midwifes, urogynaecologists and colorectal surgeons. It should be in the library of every hospital where obstetric care is provided. 